Pacific Journal of Mathematics

THE COCHRAN SEQUENCES OF SEMI-BOUNDARY LINKS 


\title{
THE COCHRAN SEQUENCES OF SEMI-BOUNDARY LINKS
}

\author{
Gyo TAEK JIN
}

\begin{abstract}
For a 1-dimensional semi-boundary link, Cochran constructed a sequence of Sato-Levine invariants of successively derived links. This is a linear recurrence sequence and conversely any linear recurrence sequence can be constructed in this way. An upper bound for the growth of this sequence is obtained.
\end{abstract}

1. Introduction. An ordered pair $L=(M, K)$ of oriented circles which are disjointly and smoothly embedded in $\mathbf{S}^{3}$ is called a semiboundary link if the linking number $\operatorname{lk}(M, K)$ is equal to zero [Sa]. If $L=(M, K)$ is a semi-boundary link, then there exist Seifert surfaces $V$ of $M$ and $W$ of $K$ which intersect only in the interiors and transversely. We call $(V, W)$ a Seifert pair for $L$.

The orientations on $V$ and $W$, which induce the orientation of $L$ on the boundaries, determine a normal 2-frame field on $V \cap W$. The Sato-Levine invariant $\beta(L)$ of the link $L$ is the homotopy class in $\pi_{3} \mathbf{S}^{2} \cong \mathbf{Z}$ which is represented by the Thom-Pontryagin construction on the 2-frame field. It is proven in [Sa] that this integer is independent of the choice of the Seifert surfaces and that it is a linkconcordance invariant. The following well-known proposition is included for completeness.

Proposition 1.1. If $L=(M, K)$ is a semi-boundary link, then

(a) $\beta(L)=0$ if $L$ is a boundary link.

(b) $\beta(\bar{L})=\beta(L)$ where $\bar{L}=(K, M)$.

(c) $\beta\left(L^{\times}\right)=\beta(L)$ where $L^{\times}$is the same as $L$ with the orientation of either $M$ or $K$ reversed.

(d) $\beta(-L)=-\beta(L)$ where $-L$ is a mirror image of $L$.

Proof. (a) is obvious by the definition of the Sato-Levine invariant. (b), (c) and (d) easily follows fromt he fact that

$$
[g \circ f \circ h]=\{\operatorname{deg}(g)\}^{2} \operatorname{deg}(h)[f]
$$

in $\pi_{3} \mathbf{S}^{2}$ where $f: \mathbf{S}^{3} \rightarrow \mathbf{S}^{2}, g: \mathbf{S}^{2} \rightarrow \mathbf{S}^{2}$ and $h: \mathbf{S}^{3} \rightarrow \mathbf{S}^{3}$ are continuous maps [Hu]. 
Let $(V, W)$ be a Seifert pair for a semi-boundary link $L=(M, K)$. We may assume that the intersection $F=V \cap W$ is connected, by a surgery on the Seifert surfaces. In this case, we call $(V, W)$ a special Seifert pair for $L$. Since $W$ is oriented, we can push a small tubular neighborhood of $F$ in $W$ off $F$ in a normal direction of $W$. Call this surface $W^{\prime}$. Then $W^{\prime}$ is a Seifert surface for $K$ such that $F \cap W^{\prime}=\varnothing$. Therefore $D(L)=(F, K)$ is a new semi-boundary link derived from $L=(M, K)$. We follow Cochran in calling $D(L)$ a derived link or a derivative of $L . D(L)$ is well defined only up to an equivalence relation called weak-cobordism which is weaker than the link-concordance [C]. Since $D(L)$ is a semi-boundary link, its SatoLevine invariant is defined. We can iterate this procedure to get a sequence of integers as follows:

(i) $\beta_{1}(L)=\beta(L)$,

(ii) $\beta_{i}(L)=\beta\left(D^{i-1}(L)\right)$, for $i \geq 2$.

This sequence $\left\{\beta_{i}(L)\right\}_{i=1}^{\infty}$ is well defined and depends only on the weak-corbordism class of $L$. We call it the Cochran sequence of $L$.

By Proposition 1.1(a), $\left\{\beta_{i}(L)\right\}_{i=1}^{\infty}$ is identically zero if $L$ is a boundary link. One of the most important properties of this sequence is that it is additive under componentwise oriented connected sum of semi-boundary links [C].

2. Kojima's function. Recall the definition of Kojima's function first given in [KY]. Let $L=(M, K)$ be a semi-boundary link and let $\widetilde{X}_{K}$ be the infinite cyclic cover of $\mathbf{S}^{3} \backslash K$ whose covering transformation group is generated by $t$. Denote by $\ell$ a zero-push-off of $M$, i.e., $\ell$ is isotopic to $M$ in a small tubular neighborhood of $M$ and $\operatorname{lk}(\ell, M)=$ 0 . Let $\tilde{\ell}$ and $\widetilde{M}$ be near-by lifts of $\ell$ and $M$ in $\widetilde{X}_{K}$. Since the Alexander polynomial $\Delta_{K}(t)$ of $K$ annihilates the $Z\left[t, t^{-1}\right]$-module $H_{1}\left(\widetilde{X}_{K}\right), \Delta_{K}(t) \tilde{\ell}$ bounds a 2 -chain $\zeta$ in $\widetilde{X}_{K}$.

DEFINITION.

$$
\eta_{L}(t)=\frac{\sum_{n=-\infty}^{\infty} \operatorname{Int}\left(\zeta, t^{n} \widetilde{M}\right) t^{n}}{\Delta_{K}(t)}
$$

where $\operatorname{Int}($,$) stands for the usual intersection number in \widetilde{X}_{K}$.

For any semi-boundary link $L=(M, K), \eta_{L}(t)$ is an invariant of $I$-equivalence class of $L$ satisfying the following properties:

(iii) $\eta_{L}\left(t^{-1}\right)=\eta_{L}(t)$,

(iv) $\eta_{L}(1)=0$.

Since $\Delta_{K}(t)$ can be normalized to satisfy $\Delta_{K}\left(t^{-1}\right)=\Delta_{K}(t)$, one can change the variable by $x=(1-t)(1-1 / t)$ to get a rational function 
$h_{L}(x)$ from $\eta_{L}(t)$. Cochran proved in [C] that $\eta_{L}(t)$ is equivalent to the sequence $\left\{\beta_{i}(L)\right\}_{i=1}^{\infty}$. The relation is given by the Maclaurin series expansion.

(v) $h_{L}(x)=\sum_{i=1}^{\infty} \beta_{i}(L) x^{i}$.

In other words, $h_{L}(x)$ is the generating function for the Cochran sequence $\left\{\beta_{i}(L)\right\}_{i=1}^{\infty}$.

\section{Linear recurrence sequences.}

Definition. An infinite sequence $\left\{n_{i}\right\}_{i=1}^{\infty}$ of integers is a linear recurrence sequence if there exist integers $N>0, a_{1}, \ldots, a_{d}$ such that

for all $i>N$.

$$
n_{i+d}+a_{1} n_{i+d-1}+\cdots+a_{d} n_{i}=0
$$

Proposition 3.1. (a) $\left\{n_{i}\right\}_{i=1}^{\infty}$ is a linear recurrence sequence if and only if its generating function is of the form

$$
\frac{b_{1} x+b_{2} x^{2}+\cdots+b_{m} x^{m}}{1+a_{1} x+a_{2} x^{2}+\cdots+a_{d} x^{d}}
$$

for some integers $d>0, m>0, a_{1}, \ldots, a_{d}$ and $b_{1}, \ldots, b_{m}$.

(b) If $\left\{n_{i}\right\}_{i=1}^{\infty}$ is a linear recurrence sequence whose generating function is as in (a), then for all sufficently large $i$,

$$
n_{i}=\sum_{j=1}^{k} P_{j}(i) \gamma_{j}^{i}
$$

where $1+a_{1} x+a_{2} x^{2}+\cdots+a_{d} x^{d}=\prod_{j=1}^{k}\left(1-\gamma_{j} x\right)^{d}$, the $\gamma_{j}$ 's are distinct, and $P_{j}(i)$ is a polynomial in $i$ of degree $d_{j}-1$.

Proposition 3.1 is a version of Theorem 4.1 in [St].

TheOREM 3.2. (a) For any semi-boundary link $L,\left\{\beta_{i}(L)\right\}_{i=1}^{\infty}$ is a linear recurrence sequence.

(b) For any linear recurrence sequence $\left\{n_{i}\right\}_{i=1}^{\infty}$, there is a semiboundary link $L$ such that $\left\{\beta_{i}(L)\right\}_{i=1}^{\infty}=\left\{n_{i}\right\}_{i=1}^{\infty}$.

Proof. (a) Using the fact that $\Delta_{K}(1)= \pm 1$, it is easy to see that $h_{L}(x)$ is of the form

$$
\frac{b_{1} x+b_{2} x^{2}+\cdots+b_{m} x^{m}}{1+a_{1} x+a_{2} x^{2}+\cdots+a_{d} x^{d}}
$$

for some integers $d>0, m>0, a_{1}, \ldots, a_{d}$ and $b_{1}, \ldots, b_{m}$. Then (a) follows from Proposition 3.2 (a) and the identity (v). 
(b) Let

$$
h(x)=\frac{b_{1} x+b_{2} x^{2}+\cdots+b_{m} x^{m}}{1+a_{1} x+a_{2} x^{2}+\cdots+a_{d} x^{d}}
$$

be the generating function for a linear recurrence sequence $\left\{n_{i}\right\}_{i=1}^{\infty}$. Substituting $(1-t)(1-1 / t)$ for $x$ in $h(x)$, we get

$$
\eta(t)=\frac{\sum_{j=1}^{m} d_{j}\left(2-t^{j}-t^{-j}\right)}{1+\sum_{i=1}^{d} c_{i}\left(2-t^{i}-t^{-i}\right)}
$$

for some integer $c_{i}$ 's and $d_{j}$ 's. We may assume that $d \geq m$ by adding terms with coefficient zero, if necessary. We will construct a semi-boundary link $L_{j}$, for each $j$ with $\varepsilon_{j} \neq 0$, satisfying

$$
\eta_{L_{j}}(t)=\frac{\varepsilon_{j}\left(2-t^{j}-t^{-j}\right)}{1+\sum_{i=1}^{d} c_{i}\left(2-t^{i}-t^{-i}\right)}
$$

where, for each $k=1, \ldots, m$,

$$
\varepsilon_{k}=\left\{\begin{aligned}
1 & \text { if } d_{k}>0 \\
0 & \text { if } d_{k}=0 \\
-1 & \text { if } d_{k}<0
\end{aligned}\right.
$$

First we will construct a knot whose Alexander polynomial is

$$
\Delta(t)=\varepsilon_{j}\left(1+\sum_{i=1}^{d} c_{i}\left(2-t^{i}-t^{-1}\right)\right)
$$

as follows [L, R1, R2].

Let $K$ be an unknot. Embed a solid torus $T$ in $\mathbf{S}^{3} \backslash K$ so that

(1) the centerline $C(T)$ of $T$ is unknotted,

(2) $\operatorname{lk}(K, C(T))=0$,

(3) there are $\varepsilon_{j} c_{1}, \ldots, \varepsilon_{j} c_{d}$ twists in $T$ separated by strands of $T$, each of which links once around $K$ as shown in Figure 1.

Let $\lambda$ be a longitude of $T$ such that $\operatorname{lk}(\lambda, C(T))=\varepsilon_{j}$ when $\lambda$ and $C(T)$ are oriented in the same direction. Since $S^{3} \backslash \operatorname{int}(T)$ is another solid torus, there is a homeomorphism

$$
h: \mathbf{S}^{\mathbf{3}} \backslash \operatorname{int}(T) \rightarrow \mathbf{S}^{\mathbf{3}} \backslash \operatorname{int}(T)
$$

sending $\lambda$ into a meridian curve $\mu$ of $T$. Let $K_{h}=h(K) \subset \mathbf{S}^{3}$ and let $\widetilde{X}$ be the infinite cyclic covering space of $\mathbf{S}^{3} \backslash K_{h}$ obtained by attaching solid tori to the infinite cyclic covering space of $S^{3} \backslash(\operatorname{int}(T) \cup K)$. Then $\Delta(t)$ is the Alexander polynomial of $K_{h}$.

Let $M$ be another unknot in $\mathbf{S}^{3} \backslash(\operatorname{int}(T) \cup K)$ which goes once around the $j$-th twist of $T$ in Figure 1. Let $M_{h}=h(M) \subset \mathbf{S}^{3}$. Then 


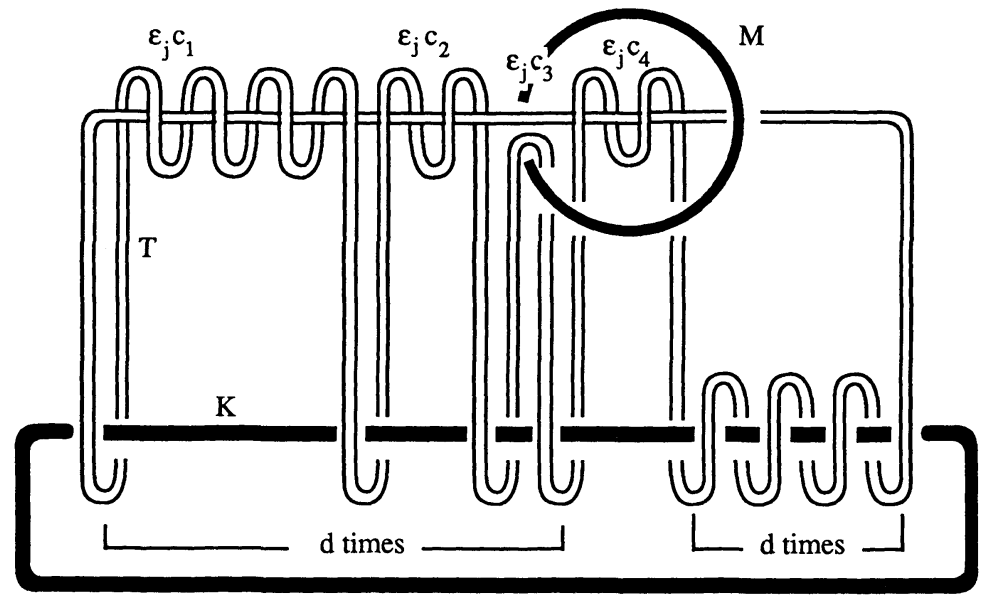

FIgURE 1. This is the case when $d=4, \varepsilon_{j} c_{1}=$ $4, \varepsilon_{j} c_{2}=-2, \varepsilon_{j} c_{3}=0, \varepsilon_{j} c_{4}=-2$ and $j=3$.

$L_{j}=\left(M_{h}, K_{h}\right)$ is a semi-boundary link. Now we compute $\eta_{L_{j}}(t)$. Choose a nonsingular disc $E$ bounded by $M$ which intersects $\partial T$ in two circles. Let $\alpha$ be one of the circles in $E \cap \partial T$. Choose a slight push-off $M^{\prime}$ of $M$ in $E$. Let $E^{\prime}$ be the disc in $E$ such that $\partial E^{\prime}=M^{\prime}$. Define $\theta=E \backslash \operatorname{int}(T)$, and $\theta^{\prime}=E^{\prime} \backslash \operatorname{int}(T)$. Let

$$
\begin{aligned}
& \widetilde{M}^{\prime} \quad \widetilde{M} \\
& \tilde{\alpha} \subset \tilde{\theta}^{\prime} \subset \tilde{\tilde{\theta}} \\
& \underset{\widetilde{E}^{\prime}}{\cap} \tilde{\widetilde{E}}
\end{aligned}
$$

be a diagram of lifts of $\alpha, \theta, \theta^{\prime}, E, E^{\prime}, M$, and $M^{\prime}$ in $\tilde{X}$. Choose a generator $t$ of the covering transformation so that

$$
\partial \tilde{\theta}^{\prime}=\widetilde{M}^{\prime}-\left(1-t^{j}\right) \tilde{\alpha}+\partial \gamma
$$

as 1-cycles in $\widetilde{X}$, where $\gamma$ is a 2-chain in $t^{j} \partial \widetilde{T}$ and $\widetilde{T}$ is a lift of $T$ containing $\tilde{\alpha}$. Since $\operatorname{lk}(K, C(T))=0$ and $K$ is unknotted, there is a singular disc $\delta$ in $\mathbf{S}^{3} \backslash K$ bounded by a 0 -longitude $\lambda_{0}$ of $T$. We may assume that $M$ and $\delta$ intersect at two points. Then there exists a lift $\tilde{\delta}$ of $\delta \backslash \operatorname{int}(T)$ in $\tilde{X}$ satisfying

$$
\operatorname{Int}\left(\tilde{\delta}, t^{n} \widetilde{M}\right)=\left\{\begin{aligned}
1 & \text { if } n=0, \\
-1 & \text { if } n=-j, \\
0 & \text { otherwise. }
\end{aligned}\right.
$$

Then it is not hard to see that

$$
\Delta(t) \tilde{\alpha}=\partial \tilde{\delta}+\partial \sigma
$$


where $\sigma$ is a 2-chain in $\bigcup_{k=-\infty}^{\infty} t^{k} \partial \tilde{T}$. Let

$$
\zeta=\left(1-t^{j}\right)(\tilde{\delta}+\sigma)+\Delta(t)\left(\tilde{\theta}^{\prime}-\gamma\right) .
$$

Then $\partial \zeta=\Delta(t) \widetilde{M}^{\prime}$. It is clear that, for any $n$,

$$
\operatorname{Int}\left(\sigma, t^{n} \widetilde{M}\right)=\operatorname{Int}\left(\tilde{\theta}^{\prime}, t^{n} \widetilde{M}\right)=\operatorname{Int}\left(\gamma, t^{n} \widetilde{M}\right)=0 .
$$

Therefore,

$$
\eta_{L}(t)=\frac{\sum_{n=-\infty}^{\infty} \operatorname{Int}\left(\left(1-t^{j}\right) \tilde{\delta}, t^{n} \widetilde{M}\right) t^{n}}{\Delta(t)}=\frac{\varepsilon_{j}\left(2-t^{j}-t^{-j}\right)}{1+\sum_{i=1}^{d} c_{i}\left(2-t^{i}-t^{-i}\right)}
$$

Then, by the identity ( $v$ ) and the additivity of the Cochran sequence,

$$
L=\underbrace{\left(L_{1} \sharp \cdots \sharp L_{1}\right)}_{\left|d_{1}\right|} \sharp \cdots \sharp \underbrace{\left(L_{m} \sharp \cdots \sharp L_{m}\right)}_{\left|d_{m}\right|}
$$

satisfies $\eta_{L}(t)=\eta(t)$. Finally, $\left\{\beta_{i}(L)\right\}_{i=1}^{\infty}=\left\{n_{i}\right\}_{i=1}^{\infty}$.

COROLlARY 3.3. (a) Any sequence of integers which eventually becomes a geometric progression is a Cochran sequence.

(b) Any sequence of integers which eventually becomes an arithmetic progresssion is a Cochran sequence.

(c) The Fibonacci sequence $\{1,1,2,3,5,8, \ldots\}$ is a Cochran sequence.

(d) For any positive integer $d,\left\{n^{d}\right\}_{n=1}^{\infty}$ is a Cochran sequence.

Proof. (a) $n_{i+1}-r n_{i}=0$ is the linear recurrence relation for a geometric progression with common ratio $r$.

(b) $n_{i+2}-2 n_{i+1}+n_{i}=0$ is the linear recurrence relation for an arithmetic progression.

(c) $n_{i+2}-n_{i+1}-n_{i}=0$ is the linear recurrence relation for the Fibonacci sequence. Figure 2 shows a link whose Cochran sequence is the Fibonacci sequence.

(d) The following identity

$$
\sum_{k=0}^{d+1}(-1)^{k}\left(\begin{array}{c}
d+1 \\
k
\end{array}\right)(n+k)^{d}=0
$$

is a linear recurrence relation for the sequence $\left\{n^{d}\right\}_{n=1}^{\infty}$.

It is obvious that Cochran sequences can be unbounded. But they cannot grow arbitrarily fast. 


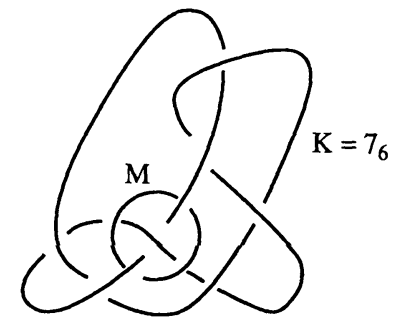

FIGURE 2. Fibonacci link

Corollary 3.4. For a semi-boundary link $L=(M, K)$, there exist constants $C>0$ and $\gamma>0$ such that

$$
\left|\beta_{n}(L)\right| \leq C n^{s} \gamma^{n}
$$

for all $n$, where $s$ is a nonnegative integer less than a half of the degree of the Alexander polynomial of $K$.

Proof. This is an easy consequence of Proposition 3.1(b).

For example, $\left\{n^{n}\right\}_{n=1}^{\infty}$ and $\{n !\}_{n=1}^{\infty}$ cannot be Cochran sequences since, for any $C, s, \gamma>0$,

$$
n^{n}>C n^{s} \gamma^{n} \text { and } n !>C n^{s} \gamma^{n}
$$

for all sufficiently large $n$.

For a semi-boundary link $L=(M, K), \bar{L}=(K, M)$ is also a semi-boundary link satisfying $\beta(\bar{L})=\beta(L)$ as in Proposition 1.1 (b). Therefore the two sequences $\left\{\beta_{i}(L)\right\}_{i=1}^{\infty}$ and $\left\{\beta_{i}(\bar{L})\right\}_{i=1}^{\infty}$ have the same first entries. By the following theorem, this is the only relation between them.

THEOREM 3.5. For any two linear recurrence sequences $\left\{n_{i}\right\}_{i=1}^{\infty}$ and $\left\{m_{i}\right\}_{i=1}^{\infty}$ such that $n_{1}=m_{1}$, there exists a semi-boundary link $L$ satisfying $\left\{\beta_{i}(L)\right\}_{i=1}^{\infty}=\left\{n_{i}\right\}_{i=1}^{\infty}$ and $\left\{\beta_{i}(\bar{L})\right\}_{i=1}^{\infty}=\left\{m_{i}\right\}_{i=1}^{\infty}$.

For a semi-boundary link $L$, and for any integer $m$, Cochran constructed a new semi-boundary link $\int_{m} L$ called an $m$-antiderivative of $L$, which is well defined up to weak-cobordism satisfying

(vi) $\beta_{1}\left(\int_{m} L\right)=m$,

(vii) $D\left(\int_{m} L\right)$ is weakly-cobordant to $L$ and hence

$$
\beta_{i}\left(\int_{m} L\right)=\beta_{i-1}(L) \text { for } i \geq 2 \text {. }
$$




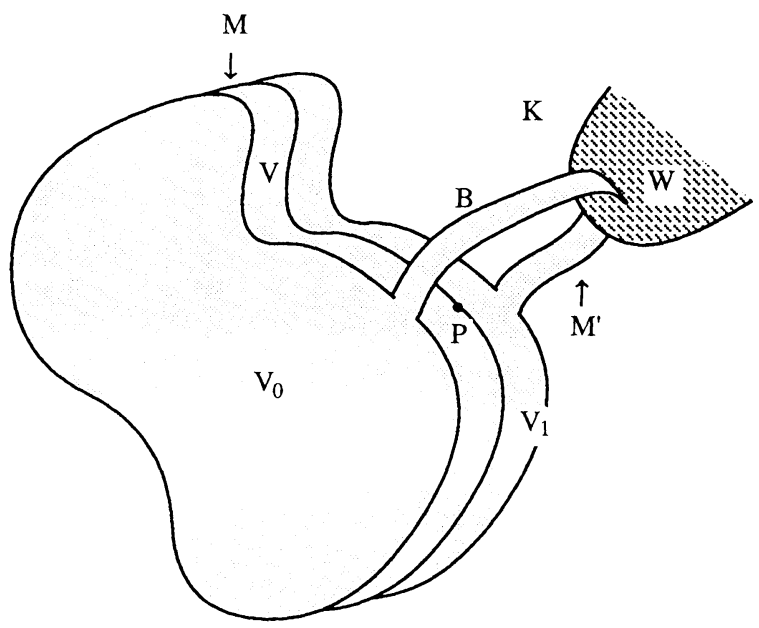

FIGURE 3

LEMMA 3.6. $D\left(\overline{\int_{0} L}\right)$ is weakly-cobordant to a boundary link for any semi-boundary link $L$.

Proof. Here we use Cochran's construction of antiderivatives. Let $L=(M, K)$ be a semi-boundary link with a special Seifert pair $(V, W)$. Let $c: V \times[0,1] \hookrightarrow \mathbf{S}^{3}$ be a collar of $V$ such that

$$
c\left(V \times\left\{\frac{1}{2}\right\}\right)=V \text { and } c(V \times[0,1]) \cap K=\varnothing .
$$

Let $V_{i}=c(V \times\{i\})$ for $i \in[0,1]$. Choose orientations on $V_{0}, V_{1}$, and $c(M \times[0,1])$ so that they agree on boundaries. In the procedure we smooth all the corners whenever necessary. Let $\gamma$ be a smooth nonsingular path joining two points $P \in M$ and $Q \in K$ such that

$$
\operatorname{int}(\gamma) \cap(c(V \times[0,1]) \cup W)=\varnothing .
$$

Let $J$ be a small closed interval on $M$ centered at $P$. Push a copy of $c(J \times(0,1))$ off $c(M \times[0,1])$ along $\gamma$ passing $Q$ slightly, so that the image of $c(J \times[0,1])$ becomes a band $B$ connecting $V_{0}$ and $V_{1}$, which intersects $\operatorname{int}(W)$ transversely on the image of $c\left(J \times\left\{\frac{1}{2}\right\}\right)$ in a small neighborhood of $Q$. See Figure 3. Then

$$
M^{\prime}=\partial(c(M \times[0,1]) \cup B)
$$

is a knot intersecting $W$ in two points. Replacing a small tubular neighborhood of $M^{\prime} \cap W$ in $W$ by a tube that goes along the lower half of $M^{\prime}$, we get a Seifert surface $W_{0}$ for $K$ such that $W_{0} \cap M^{\prime}=\varnothing$. Then

$$
\left(c(M \times[0,1]) \cup B, W_{0}\right)
$$




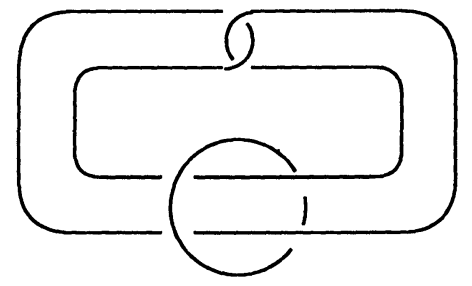

FIGURE 4. Whitehead link

is a special Seifert pair for $\left(M^{\prime}, K\right)$. It is easy to see that

$$
\left((c(M \times[0,1]) \cup B) \cap W_{0}, K\right)
$$

is ambient isotopic to $(M, K)$. Since $c(M \times[0,1])$ is untwisted, we have $\beta\left(M^{\prime}, K\right)=0$. Therefore $\left(M^{\prime}, K\right)=\int_{0} L$. Using the same Seifert surfaces for $\overline{\int_{0} L}=\left(K, M^{\prime}\right)$, we get $D\left(\overline{\int_{0} L}\right)=\left(M, M^{\prime}\right)$. Since $V \cap\left(V_{0} \cup V_{1} \cup B\right)=\varnothing$,

$$
\left(M, M^{\prime}\right)=\left(\partial V, \partial\left(V_{0} \cup V_{1} \cup B\right)\right)
$$

is a boundary link.

COROLlaRY 3.7. $\left\{\beta_{i}\left(\overline{\int_{0} L}\right)\right\}_{i=1}^{\infty}$ is identically zero.

Proof of Theorem 3.5. By Theorem 3.2, there are semi-boundary links $L_{1}$ and $L_{2}$ such that $\left\{\beta_{i}\left(L_{1}\right)\right\}_{i=1}^{\infty}=\left\{n_{j}\right\}_{j=2}^{\infty}$ and $\left\{\beta_{i}\left(L_{2}\right)\right\}_{i=1}^{\infty}=$ $\left\{m_{j}\right\}_{j=2}^{\infty}$.

Let $W$ be the Whitehead link as in Figure 4 . Then it is easy to see that

$$
\left\{\beta_{i}(W)\right\}_{i=1}^{\infty}=\left\{\beta_{i}(\bar{W})\right\}_{i=1}^{\infty}=\{1,0,0, \ldots\}
$$

and

$$
\left\{\beta_{i}(-W)\right\}_{i=1}^{\infty}=\left\{\beta_{i}(\overline{-W})\right\}_{i=1}^{\infty}=\{-1,0,0, \ldots\}
$$

where $-W$ is the mirror image of $W$. Let

$$
H=\left\{\begin{array}{cl}
W & \text { if } n_{1} \geq 0 \\
-W & \text { if } n_{1}<0
\end{array}\right.
$$

Define

$$
L=\underbrace{H \sharp \cdots \sharp H}_{\left|n_{1}\right|} \sharp \int_{0} L_{1} \sharp \overline{\int_{0} L_{2}} .
$$


Then, by the additivity of the Cochran sequence and Corollary 3.7, we have

$$
\begin{aligned}
\beta_{i}(L) & =\left|n_{1}\right| \beta_{i}(H)+\beta_{i}\left(\int_{0} L_{1}\right)+\beta_{i}\left(\overline{\int_{0} L_{2}}\right) \\
& = \begin{cases}n_{1}+0+0 & \text { if } i=1, \\
0+n_{i}+0 & \text { if } i>1\end{cases} \\
& =n_{i} \quad \text { for all } i .
\end{aligned}
$$

Similarly,

$$
\begin{aligned}
\beta_{i}(\bar{L}) & =\left|n_{1}\right| \beta_{i}(\bar{H})+\beta_{i}\left(\overline{\int_{0} L_{1}}\right)+\beta_{i}\left(\int_{0} L_{2}\right) \\
& = \begin{cases}m_{1}+0+0 & \text { if } i=1, \\
0+0+m_{i} & \text { if } i>1\end{cases} \\
& =m_{i}, \quad \text { for all } i .
\end{aligned}
$$

Acknowledgment. The author gives thanks to his thesis advisor Jerome Levine without whom this work would not have been done.

\section{REFERENCES}

[C] T. Cochran, Geometric invariants of link cobordism, Commentarii Math. Helv., 60 (1985), 291-311.

[F] R. A. Fenn, Techniques of Geometic Topology, London Math. Soc. Lecture Note Ser., 57 (1983).

[Hu] D. Husemoller, Fibre Bundles, New York: Springer-Verlag 1975.

[J] G. T. Jin, Invariants of two-component links, Ph.D. Thesis, Brandeis University, 1988.

[KY] S. Kojima and M. Yamasaki, Some new invariants of links, Inventiones Math., 54 (1979), 213-228.

[L] J. Levine, A characterization of knot polynomials, Topology, 4 (1965), 135141.

[R1] D. Rolfsen, A surgical view of Alexander's polynomial, Geometric Topology, Lecture Notes in Math., vol. 438, Springer-Verlag, 1974, 415-423.

[R2] _ Knots and Links, Berkeley: Publish or Perish, 1976.

[Sa] N. Sato, Cobordism of semi-boundary links, Topology Appl., 18 (1984), 225234.

[St] R. P. Stanley, Generating functions, Studies in Combinatorics, Studies in Mathematics, 17 (1978), 100-141.

Received December 9, 1989 and in revised form April 18, 1990.

KoREA INSTITUTE OF TeChNOLOGY

TAEJON, Korea 305-701 


\section{PACIFIC JOURNAL OF MATHEMATICS EDITORS}

\author{
V. S. VARAdaraJan \\ (Managing Editor) \\ University of California \\ Los Angeles, CA 90024-1555-05 \\ Herbert Clemens \\ University of Utah \\ Salt Lake City, UT 84112 \\ THOMAS ENRIGHT \\ University of California, San Diego \\ La Jolla, CA 92093
}

R. FINN

Stanford University

Stanford, CA 94305

Hermann FlaschKa

University of Arizona

Tucson, AZ 85721

VAUGHAN F. R. Jones

University of California

Berkeley, CA 94720

STEVEN KerckHofF

Stanford University

Stanford, CA 94305

\section{C. MOORE}

University of California

Berkeley, CA 94720

Martin ScharlemanN

University of California

Santa Barbara, CA 93106

HAROLd STARK

University of California, San Diego

La Jolla, CA 92093
R. ARENS
E. F. BECKENBACH
F. WoLF
K. YoshidA
ASSOCIATE EDITORS

\begin{tabular}{ll}
\multicolumn{1}{c}{$(1906-1982)$} & \multicolumn{1}{c}{$(1904-1989)$} \\
SUPPORTING & INSTITUTIONS \\
UNIVERSITY OF ARIZONA & UNIVERSITY OF OREGON \\
UNIVERSITY OF BRITISH COLUMBIA & UNIVERSITY OF SOUTHERN CALIFORNIA \\
CALIFORNIA INSTITUTE OF TECHNOLOGY & STANFORD UNIVERSITY \\
UNIVERSITY OF CALIFORNIA & UNIVERSITY OF HAWAII \\
MONTANA STATE UNIVERSITY & UNIVERSITY OF TOKYO \\
UNIVERSITY OF NEVADA, RENO & UNIVERSITY OF UTAH \\
NEW MEXICO STATE UNIVERSITY & WASHINGTON STATE UNIVERSITY \\
OREGON STATE UNIVERSITY & UNIVERSITY OF WASHINGTON
\end{tabular}

The Supporting Institutions listed above contribute to the cost of publication of this Journal, but they are not owners or publishers and have no responsibility for its content or policies.

Mathematical papers intended for publication in the Pacific Journal of Mathematics should be in typed form or offset-reproduced (not dittoed), double spaced with large margins. Please do not use built up fractions in the text of the manuscript. However, you may use them in the displayed equations. Underline Greek letters in red, German in green, and script in blue. The first paragraph must be capable of being used separately as a synopsis of the entire paper. In particular it should contain no bibliographic references. Please propose a heading for the odd numbered pages of less than 35 characters. Manuscripts, in triplicate, may be sent to any one of the editors. Please classify according to the 1991 Mathematics Subject Classification scheme which can be found in the December index volumes of Mathematical Reviews. Supply name and address of author to whom proofs should be sent. All other communications should be addressed to the managing editor, or Elaine Barth, University of California, Los Angeles, California 90024-1555-05.

There are page-charges associated with articles appearing in the Pacific Journal of Mathematics. These charges are expected to be paid by the author's University, Government Agency or Company. If the author or authors do not have access to such Institutional support these charges are waived. Single authors will receive 50 free reprints; joint authors will receive a total of 100 free reprints. Additional copies may be obtained at cost in multiples of 50 .

The Pacific Journal of Mathematics (ISSN 0030-8730) is published monthly except for July and August. Regular subscription rate: $\$ 190.00$ a year (10 issues). Special rate: $\$ 95.00$ a year to individual members of supporting institutions.

Subscriptions, orders for numbers issued in the last three calendar years, and changes of address should be sent to Pacific Journal of Mathematics, P.O. Box 969, Carmel Valley, CA 93924, U.S.A. Old back numbers obtainable from Kraus Periodicals Co., Route 100, Millwood, NY 10546.

The Pacific Journal of Mathematics at P.O. Box 969, Carmel Valley, CA 93924 (ISSN 0030-8730) is published monthly except for July and August. Second-class postage paid at Carmel Valley, California 93924, and additional mailing offices. Postmaster: send address changes to Pacific Journal of Mathematics, P.O. Box 969, Carmel Valley, CA 93924.

PUBLISHED BY PACIFIC JOURNAL OF MATHEMATICS, A NON-PROFIT CORPORATION Copyright (C) 1991 by Pacific Journal of Mathematics 


\section{Pacific Journal of Mathematics}

Vol. 149, No. $2 \quad$ June, 1991

Manuel Alfaro Garcia, Mark Conger and Kenneth Hodges, The structure

of singularities in $\Phi$-minimizing networks in $\mathbf{R}^{2}$

Werner Balser, Dependence of differential equations upon parameters in

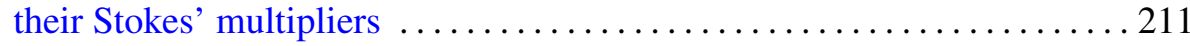

Enrico Casadio Tarabusi and Stefano Trapani, Envelopes of holomorphy

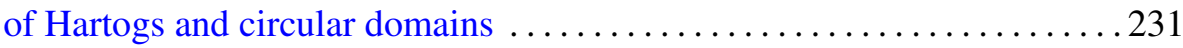

Hermann Flaschka and Luc Haine, Torus orbits in $G / P \ldots \ldots \ldots \ldots 251$

Gyo Taek Jin, The Cochran sequences of semi-boundary links .......... 293

Yasuyuki Kawahigashi, Cohomology of actions of discrete groups on

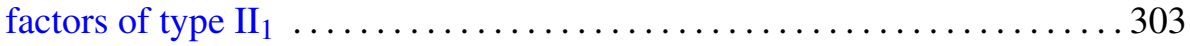

Ki Hyoung Ko and Lawrence Smolinsky, A combinatorial matrix in 3-manifold theory

W. B. Raymond Lickorish, Invariants for 3-manifolds from the combinatorics of the Jones polynomial

Peter Arnold Linnell, Zero divisors and group von Neumann algebras . . . . . 349

Bruce Harvey Wagner, Classification of essential commutants of abelian

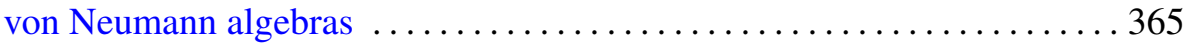

Herbert Walum, Multiplication formulae for periodic functions ..........383 